\title{
BROWNIAN MOTION AND POLYMER STATISTICS ON CERTAIN CURVED MANIFOLDS
}

\author{
Radu P. Mondescut and M. Muthukumar \\ Department of Physics \& Astronomy, and Polymer Science $\&$ Engineering Department and Materials Research Science and \\ Engineering Center, \\ University of Massachusetts, Amherst, MA 01003
}

\begin{abstract}
In this paper we have considered a Gaussian polymer chain of length $L$ as an intrinsic object enclosed on a surface embedded in the Euclidean space. When the surfaces are the sphere $\mathrm{S}^{D-1}$ in $D$ dimensions and the cylinder, the cone and the curved torus in $\mathbb{R}^{3}$, we have calculated analytically and numerically (using the diffusion equation and the path-integral approach) the probability distribution function $\mathrm{G}\left(\mathbf{R} \mid \mathbf{R}^{\prime} ; L\right)$ of the end-to-end vector $\mathbf{R}-\mathbf{R}^{\prime}$ and the mean-square end-to-end distance $\left\langle\left(\mathbf{R}-\mathbf{R}^{\prime}\right)^{2}\right\rangle$ of the polymer chain. Our findings are: the curvature of the surfaces induces a geometrical localization area; at short scales $(L \rightarrow 0)$ the polymer is locally flat and the mean-square end-to-end distance is just the Gaussian value $L l(l=\mathrm{Kuhn}$ length), independent of the metric properties of the surface; at large scales $(L \rightarrow \infty),\left\langle\left(\mathbf{R}-\mathbf{R}^{\prime}\right)^{2}\right\rangle$ tends to a constant value in the sphere case, it is linear in $L$ for the cylinder and reaches different constant values (as a function of the geometry of the surface) for the curved torus. In the case of the cone, contraction of the chain is induced at all length scales by the presence of the vertex, as a function of the opening angle $2 \alpha$ and the end position $\mathbf{R}^{\prime}$ of the chain. Explicit crossover formulas are derived for $G\left(\mathbf{R} \mid \mathbf{R}^{\prime} ; L\right)$ and $\left\langle\left(\mathbf{R}-\mathbf{R}^{\prime}\right)^{2}\right\rangle$.
\end{abstract}

\section{INTRODUCTION}

Statistics and topological characteristics of polymers embedded in curved interfaces are of significance in biological problems related to the way polymers wrap around spherical and rodlike macromolecules and vesicles, and the role played by geometry and topology in the dynamics of a biomolecule captured on the membrane of a cell [1]. These types of issues arise in other area of physics as well: the quantum mechanics of free particles in curved and multiply connected spaces (e.g. see Ref. [2]), the physics of vortex lines in Type II superconductors [3] and the rotational Brownian motion.

In realistic situations, excluded-volume interaction between polymer segments and fluctuations of curved interfaces need to be accounted for. Under these circumstances, only approximate analytical results can be generally obtained via perturbation analysis, variational techniques or renormalization group theory. Before embarking on such an effort, it is necessary to solve the problem of a free polymer chain, characterized only by its connectivity, on a curved, fixed surface.

In the present paper we derive, using path-integral and spectral representation, the probability distribution of the end-to-end vector $\mathbf{G}\left(\mathbf{R} \mid \mathbf{R}^{\prime} ; L\right)$ and the size - expressed by the mean-square end-to-end distance $\left\langle\left(\mathbf{R}-\mathbf{R}^{\prime}\right)^{2}\right\rangle$ in the ambient space - of an ideal (noninteracting) Gaussian polymer lying on a curved manifold.
The curved surfaces studied in this paper are the $\mathrm{S}^{D-1}$ sphere in $D$ dimensions (Fig. 11 A, for $D=3$ ); the cylinder (Fig. 1B), the circular cone (Fig. 17C) and the torus (Fig. 1D) in $\mathbb{R}^{3}$. The geometrical parameters are shown in Fig. 1. The polymer is an intrinsic $D-1$-dimensional object in the spherical surface, while it is 2 -dimensional in the other surfaces.

Although, due to the equivalence between the randomwalk problem and the statistics of an ideal Gaussian chain [4, many results are already available from the theory of Brownian motion, the specific problems we address here have not been answered yet.

In our calculations we have used the path-integral representation [4] of the probability distribution function $\mathrm{G}\left(\mathbf{R} \mid \mathbf{R}^{\prime} ; L\right)$ of a Gaussian polymer chain and the diffusion equation obeyed by this probability. As mentioned, no excluded volume or other types of interactions are accounted for in this paper.

\section{FORMULATION}

Let us consider a $D$-dimensional Gaussian polymer chain with $N$ links of Kuhn-length $l$ and total length $L=N l$, with the first bead located at $\mathbf{R}^{\prime}$ and the last one at $\mathbf{R}$. The end-to-end vector $\mathbf{R}-\mathbf{R}^{\prime}$ is distributed according to the following probability distribution function, given as an explicit path integral [5] 


$$
\begin{aligned}
\mathrm{G}\left(\mathbf{R} \mid \mathbf{R}^{\prime} ; L\right) & =\lim _{\substack{N \rightarrow \infty \\
\delta s \rightarrow 0 \\
N \delta s=L}}\left(\frac{D}{2 \pi l \delta s}\right)^{\frac{D N}{2}} \int_{\mathbf{r}_{0}=\mathbf{R}^{\prime}}^{\mathbf{r}_{N}=\mathbf{R}} \prod_{j=1}^{N-1} d^{D} \mathbf{r}_{j} \\
& \times \exp \left[-\frac{D}{2 l \delta s} \sum_{j=1}^{N}\left(\mathbf{r}_{j}-\mathbf{r}_{j-1}\right)^{2}\right],
\end{aligned}
$$

where $D$ is the intrinsic dimension of the polymer, $\delta s$ is the change in the arc-length $s$ along the chain and $\mathbf{r}_{j}$ is the position vector of the $j$-th bead of the polymer chain. Note that the distance on the surface is calculated as $\left(\mathbf{r}_{j}-\mathbf{r}_{j-1}\right)^{2}=g_{u v}\left(u_{j}-u_{j-1}\right)\left(v_{j}-v_{j-1}\right)$, where $u, v$ are the intrinsic coordinates and the metric tensor $g_{u v}$ is discretized in a symmetric form or using the midpoint rule (e.g. see Ref. [ [6.7]]). In $\mathbb{R}^{D}$, the expression (2.1) gives the Gaussian probability distribution:

$$
\mathrm{G}\left(\mathbf{R} \mid \mathbf{R}^{\prime} ; L\right)=\left(\frac{D}{2 \pi L l}\right)^{\frac{D}{2}} \exp \left[-\frac{D}{2 L l}\left(\mathbf{R}-\mathbf{R}^{\prime}\right)^{2}\right]
$$

The probability $\mathrm{G}\left(\mathbf{R} \mid \mathbf{R}^{\prime} ; L\right)$ obeys the diffusion equation

$$
\left(\frac{\partial}{\partial L}-\frac{l}{2 D} \Delta\right) \mathrm{G}\left(\mathbf{R} \mid \mathbf{R}^{\prime} ; L\right)=0
$$

subject to the initial condition

$$
\lim _{L \rightarrow 0} \mathrm{G}\left(\mathbf{R} \mid \mathbf{R}^{\prime} ; L\right)=\delta^{(D)}\left(\mathbf{R}-\mathbf{R}^{\prime}\right) .
$$

When the polymer lies in a curved manifold, one needs to employ the Laplace-Beltrami operator given by (e.g. see Ref. [ [6]])

$$
\Delta=\frac{1}{\sqrt{g}} \partial_{i} g^{i j} \partial_{j}
$$

where $g^{i j}$ is the inverse of the metric tensor $g_{i j}$ of the surface and $g=\operatorname{det}\left(g_{i j}\right)$. We remark that $g_{i j}$ is the induced metric from the ambient embedding space $\left(\mathbb{R}^{D}\right.$ or $\left.\mathbb{R}^{3}\right)$.

One can obtain the solution of Eq. (2.3) satisfying the initial condition (2.4) by applying a Laplace transform with respect to $L$ that gives the Green's function equation

$$
\left(E-\frac{l}{2 D} \Delta\right) \widetilde{\mathrm{G}}\left(\mathbf{R} \mid \mathbf{R}^{\prime} ; E\right)=\delta^{(D)}\left(\mathbf{R}-\mathbf{R}^{\prime}\right),
$$

which can be solved by an eigenfunction expansion of $\widetilde{G}$ followed by an inverse Laplace transform that eventually yields the heat kernel:

$$
\mathrm{G}\left(\mathbf{R} \mid \mathbf{R}^{\prime} ; L\right)=\sum_{\mu} \phi_{\mu}(\mathbf{R}) \phi_{\mu}^{*}\left(\mathbf{R}^{\prime}\right) \exp \left(-E_{\mu} L\right) .
$$

Here $\phi_{\mu}$ and $E_{\mu}$ are obtained from the eigenvalue equation

$$
-\frac{l}{2 D} \Delta \phi_{\mu}(\mathbf{R})=E_{\mu} \phi_{\mu}(\mathbf{R})
$$

with $\mu$ some generalized index. In general, the probability distribution $\mathrm{G}\left(\mathbf{R} \mid \mathbf{R}^{\prime} ; L\right)$ is not translational invariant. The mean-square end-to-end distance can be calculated from

$$
\left\langle\left(\mathbf{R}-\mathbf{R}^{\prime}\right)^{2}\right\rangle=\frac{\int d^{D} \mathbf{R} d^{D} \mathbf{R}^{\prime}\left(\mathbf{R}-\mathbf{R}^{\prime}\right)^{2} \mathrm{G}\left(\mathbf{R} \mid \mathbf{R}^{\prime} ; L\right)}{\int d^{D} \mathbf{R} d^{D} \mathbf{R}^{\prime} \mathrm{G}\left(\mathbf{R} \mid \mathbf{R}^{\prime} ; L\right)} .
$$

It is to be noted that the calculated mean-square distance represents the distance between the ends of the polymer measured by an outside observer, located in the ambient space, which is different from the geodesic separation measured along the surface.

\section{CALCULATIONS AND RESULTS}

Due to the known equivalence 4,8 , between the diffusion equation (2.3) and the Schrödinger equation for a free particle, we can readily obtain the polymer probability distribution $\mathrm{G}\left(\mathbf{R}, \mathbf{R}^{\prime} ; L\right)$ for the sphere and the cylinder cases, from the corresponding probability amplitudes for a rigid rotor in $D$ dimensions [9] and for a $2-D$ free particle moving on the surface of a cylinder, respectively.

\section{A. The Sphere $\mathbf{S}^{D-1}$ in $\mathbb{R}^{D}$}

Let $a$ be the radius of the $D-1$ dimensional sphere centered at the origin of the coordinate system. Then $\mathbf{R}(s)$ is a vector of magnitude $a$ that measures the position of the bead at $s$ about the center of the sphere. The intrinsic dimension of the polymer is $D-1$. In spherical coordinates the Laplace-Beltrami operator (2.5) becomes [7, $\S 4.2 .3]$

$$
\Delta=\frac{1}{a^{2}} \mathcal{L}^{2}
$$

where $\mathcal{L}^{2}$ is the $D$-dimensional Legendre operator, satisfying the eigenvalue equation

$$
\mathcal{L}^{2} \mathrm{~S}_{J}^{m}(\Omega)=-J(J+D-2) \mathrm{S}_{J}^{m}(\Omega) .
$$

The $S_{I}^{m}$ functions are the hyperspherical harmonics orthonormal and complete on the unit $\mathrm{S}^{D-1}$ sphere [10]: 


$$
\begin{aligned}
\int d \Omega \mathrm{S}_{J}^{m}(\Omega) \mathrm{S}_{J^{\prime}}^{m^{\prime}}(\Omega) & =\delta_{J J^{\prime}} \delta_{m m^{\prime}} \\
\sum_{J=0}^{\infty} \sum_{m=1}^{M} \mathrm{~S}_{J}^{m}(\Omega) \mathrm{S}_{J}^{m *}\left(\Omega^{\prime}\right) & =\delta^{(D)}\left(\Omega-\Omega^{\prime}\right),
\end{aligned}
$$

where $M=\frac{(2 J+D-2)(J+D-3) !}{J !(D-2) !}$ and $\Omega$ is the solid angle in $D$ dimensions. Using Eq. (3.2) and the eigenvalue expansion (2.7), where $\phi_{\mu} \rightarrow \mathrm{S}_{J}^{m}$ and $E_{\mu} \rightarrow J(J+D-2)$, we readily find the probability distribution function of a polymer chain (or equivalently, of a random walk) that starts at $\mathbf{R}^{\prime}$ and ends at $\mathbf{R}$ in $N=L / l$ steps, on the $\mathrm{S}^{D-1}$ sphere:

$$
\begin{aligned}
\mathrm{G}\left(\mathbf{R} \mid \mathbf{R}^{\prime} ; L\right) & =\frac{1}{a^{D-1}} \sum_{J=0}^{\infty} \sum_{m=1}^{M} \mathrm{~S}_{J}^{m}(\Omega) \mathrm{S}_{J}^{m *}\left(\Omega^{\prime}\right) \\
& \times \exp \left[-\frac{L l}{2 a^{2}} \frac{J(J+D-2)}{D-1}\right] .
\end{aligned}
$$

The probability found above is normalized properly to one $\left(\int d^{D} \mathbf{R} \mathrm{G}\left(\mathbf{R} \mid \mathbf{R}^{\prime} ; L\right)=1\right)$. In $\mathbb{R}^{3}$ we recover the known [11) rotational diffusion result:

$$
\begin{aligned}
\mathrm{G}\left(\mathbf{R} \mid \mathbf{R}^{\prime} ; L\right) & =\frac{1}{a^{2}} \sum_{l=0}^{\infty} \sum_{m=-l}^{l} \mathrm{Y}_{l m}(\Omega), \mathrm{Y}_{l m}^{*}\left(\Omega^{\prime}\right) \\
& \times \exp \left[-\frac{L l}{4 a^{2}} J(J+1)\right] .
\end{aligned}
$$

Because $\mathrm{G}\left(\mathbf{R} \mid \mathbf{R}^{\prime} ; L\right)$ is translationally invariant, the endto-end distance can be calculated as a conditional expectation value by dropping the integration over $\mathbf{R}^{\prime}$ in Eq. (2.9). To evaluate the remaining integral, we use the addition theorem for hyper-spherical harmonics 10]

$$
\begin{aligned}
\sum_{m=1}^{M} \mathrm{~S}_{J}^{m}(\Omega) \mathrm{S}_{J}^{m *}\left(\Omega^{\prime}\right) & =\frac{1}{S_{D}} \frac{(2 J+D-2)}{D-2} \\
& \times C_{J}^{(D-2) / 2}\left(\cos \Psi_{\mathbf{R R}^{\prime}}\right),
\end{aligned}
$$

where $S_{D}=\frac{2 \pi^{D / 2}}{\Gamma(D / 2)}$ and $C_{J}^{\nu}$ are the Gegenbauer polynomials of argument $\cos \psi_{\mathbf{R R}^{\prime}}$. Writing $\left(\mathbf{R}-\mathbf{R}^{\prime}\right)^{2}=$ $2 a^{2}\left[1-\cos \Psi_{\mathbf{R R}^{\prime}}\right]$ and inserting (3.4) in (2.9), we get:

$$
\begin{aligned}
\left\langle\left(\mathbf{R}-\mathbf{R}^{\prime}\right)^{2}\right\rangle & =2 a^{2}\left(1-\frac{1}{S_{D}} \sum_{J=0}^{\infty} \int d \Omega \frac{2 J+D-2}{D-2}\right. \\
& \times \cos \Psi_{\mathbf{R R}^{\prime}} C_{J}^{(D-2) / 2}\left(\cos \Psi_{\mathbf{R R}^{\prime}}\right) \\
& \left.\times \exp \left[-\frac{L l}{2 a^{2}} \frac{J(J+D-2)}{D-1}\right]\right) .
\end{aligned}
$$

Choosing $\mathbf{R}^{\prime}$ along one of the coordinate axes and applying the recurrence relation [12, $\S 8.933]$

$$
\begin{aligned}
\cos \theta C_{l}^{\nu}(\cos \theta) & =\frac{1}{2(l+\nu)}\left[(l+1) C_{l+1}^{\nu}(\cos \theta)\right. \\
& \left.+(2 \nu+l-1) C_{l-1}^{\nu}(\cos \theta)\right],
\end{aligned}
$$

we notice that the orthogonality of the Gegenbauer polynomials (with $C_{0}^{\nu}(\cos \theta)=1$ ) implies that all terms except $J=1$ are zero. From (3.7) we finally obtain the mean-square end-to-end vector for a polymer living on the surface of a $D-1$-dimensional sphere as:

$$
\begin{aligned}
\left\langle\left(\mathbf{R}-\mathbf{R}^{\prime}\right)^{2}\right\rangle & =2 a^{2}\left[1-\exp \left(-\frac{L l}{2 a^{2}}\right)\right] \\
& \simeq\left\{\begin{array}{l}
L l ; L l \ll a^{2} . \\
2 a^{2} ; L l \gg a^{2} .
\end{array}\right.
\end{aligned}
$$

Therefore, a characteristic area $2 a^{2}$ called geometrical localization area emerges in the description of $\left\langle\left(\mathbf{R}-\mathbf{R}^{\prime}\right)^{2}\right\rangle$, due to the geometrical restriction of space.

In contrast to the problem of a stiff polymer chain without any confinement, where the chain expands due to chain stiffness, chain contracts here by geometrical confinement. If the area of the confining sphere is small in comparison with $L l$, then the polymer wraps many times around the sphere so that $\left\langle\left(\mathbf{R}-\mathbf{R}^{\prime}\right)^{2}\right\rangle$ approaches the geometrical localization area. On the other hand if $a^{2} \gg L l$, then the polymer does not sense the curvature of the sphere so that $\left\langle\left(\mathbf{R}-\mathbf{R}^{\prime}\right)^{2}\right\rangle=L l$. The meansquare end-to-end distance (3.9) normalized at $a^{2}$ is plotted in Fig. 14 as a function of the dimensionless variable $\frac{L l}{a^{2}}$. It is also to be noted that $\left\langle\left(\mathbf{R}-\mathbf{R}^{\prime}\right)^{2}\right\rangle$ is independent of $D$.

\section{B. The Cylinder in $\mathbb{R}^{3}$}

Consider a cylinder of radius $a$ and of infinite length, with the induced metric from $\mathbb{R}^{3}$ (in cylindrical coordinates, with $O z$ along the axis of the cylinder): $g_{\phi \phi}=a^{2}$; $g_{\phi z}=0 ; g_{z z}=1 ; \sqrt{g}=a$. The position vector $\mathbf{R}(s)$ of any bead $s$ has the components $(a \cos \phi, a \sin \phi, z)$. The polymer dimension is $D=2$.

The Green's function equation in the Laplace space (2.6) can be solved directly by expanding the $\widetilde{\mathrm{G}}$ function in the complete set of orthogonal functions on the circle $e^{i m\left(\phi-\phi^{\prime}\right)}$ and Fourier transforming with respect to $z-z^{\prime}$, which yields

$$
\begin{aligned}
\widetilde{\mathrm{G}}\left(\mathbf{R} \mid \mathbf{R}^{\prime} ; E\right) & =\frac{1}{2 \pi a} \sum_{m=-\infty}^{\infty} \int_{-\infty}^{+\infty} \frac{d k}{2 \pi} \frac{1}{E+\frac{l m^{2}}{4 a^{2}}+\frac{l k^{2}}{4}} \\
& \times e^{i m\left(\phi-\phi^{\prime}\right)} e^{i k\left(z-z^{\prime}\right)}
\end{aligned}
$$

After applying an inverse Laplace transform and evaluating the integral over $k$, the spectral expansion of $\mathrm{G}\left(\mathbf{R} \mid \mathbf{R}^{\prime} ; L\right)$ is found to be 


$$
\begin{aligned}
\mathrm{G}\left(\mathbf{R} \mid \mathbf{R}^{\prime} ; L\right) & =\frac{1}{2 \pi a} \sqrt{\frac{1}{\pi L l}} e^{-\frac{\left(z-z^{\prime}\right)^{2}}{L l}} \\
& \times \sum_{m=-\infty}^{\infty} e^{i m\left(\phi-\phi^{\prime}\right)} e^{-\frac{L l m^{2}}{4 a^{2}}}
\end{aligned}
$$

An equivalent form of the expression above can be obtained by using the relation

$$
\exp \left(-\lambda x^{2}\right)=\frac{1}{\sqrt{2 \pi}} \int_{-\infty}^{+\infty} d \xi e^{-\frac{1}{2} \xi^{2}+i \sqrt{2 \lambda} \xi x} \quad(\lambda>0)
$$

and the Poisson formula

$$
\sum_{m=-\infty}^{+\infty} e^{2 i \pi \alpha m}=\sum_{n=-\infty}^{+\infty} \delta(\alpha-n), \quad(m, n \in \mathbb{Z}) .
$$

Applying these transformations in Eq. (3.11), we arrive at the the winding number expansion

$$
\mathrm{G}\left(\mathbf{R} \mid \mathbf{R}^{\prime} ; L\right)=\frac{1}{\pi L l} e^{-\frac{\left(z-z^{\prime}\right)^{2}}{L l}} \sum_{n=-\infty}^{\infty} e^{-\frac{a^{2}\left(\phi-\phi^{\prime}+2 \pi n\right)}{L l}},
$$

where $n$ (a topological term) is the winding number that counts how many times the chain winds around the cylinder. A term in the sum represents now the probability that a given chain starting at $\mathbf{R}$ and ending at $\mathbf{R}^{\prime}$ will wind around the cylinder $n$ times (in $L / l$ steps). This expansion can be used to investigate the statistics of the winding number (e.g. the mean-square winding number $\left.\left\langle N^{2}\right\rangle\right)$.

To calculate the mean-square end-to-end vector, we insert $\left(\mathbf{R}-\mathbf{R}^{\prime}\right)^{2}=\left(z-z^{\prime}\right)^{2}+2 a^{2}\left[1-\cos \left(\phi-\phi^{\prime}\right)\right]$ and the distribution function (3.11) in (2.9). Evaluating the integrals, we get

$$
\begin{aligned}
\left\langle\left(\mathbf{R}-\mathbf{R}^{\prime}\right)^{2}\right\rangle & =\frac{L l}{2}+2 a^{2}\left[1-e^{-\frac{L l}{4 a^{2}}}\right] \\
& \simeq \begin{cases}L l ; L l \ll a^{2} . \\
\frac{L l}{2} ; L l \gg a^{2} .\end{cases}
\end{aligned}
$$

As expected, the polymer remains almost Gaussian (a prefactor of $1 / 2$ instead of 1 ) along the $O z$ direction. The influence of the geometry appears through the radius $a$, related to the mean curvature of the cylinder. Once again, the polymer becomes geometrically localized. The mean-square end-to-end distance (normalized at $a^{2}$ ) is represented in Fig. 1 as a function of the dimensionless variable $\frac{L l}{a^{2}}$.

\section{The Cone in $\mathbb{R}^{3}$}

Now let us consider the surface of cone with the vertex at the origin $O$, centered about the $O z$ axis and with the opening angle $2 \alpha$. In cylindrical coordinates, the position vector $\mathbf{R}(s)$ of any point $s$ along the chain is $\mathbf{R}(s)=(\rho(s) \cos \phi(s), \rho(s) \sin \phi(s), \rho(s) \cot \alpha)$ and the induced metric is: $g_{\rho \rho}=1+\cot ^{2} \alpha ; g_{\rho \phi}=0 ; g_{\phi \phi}=\rho^{2}$; $\sqrt{g}=\rho \sqrt{1+\cot ^{2} \alpha}$. As in the previous problem, the dimension of the polymer is $D=2$.

To compute the probability distribution $\mathrm{G}\left(\mathbf{R} \mid \mathbf{R}^{\prime} ; L\right)$ of the end-to-end vector we start with the Green's function equation (2.6) obeyed by the Laplace transform $\widetilde{\mathrm{G}}$ :

$$
\begin{aligned}
& \left(E-\frac{l}{4 \rho} \sin ^{2} \alpha \frac{\partial}{\partial \rho} \rho \frac{\partial}{\partial \rho}-\frac{l}{4 \rho^{2}} \frac{\partial^{2}}{\partial \phi^{2}}\right) \widetilde{G}\left(\mathbf{R} \mid \mathbf{R}^{\prime} ; E\right) \\
& =\frac{\sin \alpha}{\rho} \delta\left(\rho-\rho^{\prime}\right) \delta\left(\phi-\phi^{\prime}\right)
\end{aligned}
$$

We solve this equation in a standard manner [13]. First we eliminate the azimuthal dependence of $\widetilde{G}$ by inserting the expansion

$$
\begin{aligned}
\widetilde{\mathrm{G}}\left(\mathbf{R} \mid \mathbf{R}^{\prime} ; E\right) & =\frac{1}{2 \pi} \sum_{m=-\infty}^{+\infty} \tilde{\mathrm{g}}_{m}\left(\rho \mid \rho^{\prime} ; E\right) \\
& \times \exp \left[i m\left(\phi-\phi^{\prime}\right)\right],
\end{aligned}
$$

in $(3.16)$, which gives

$$
\begin{aligned}
& {\left[\frac{1}{x} \frac{\partial}{\partial x} x \frac{\partial}{\partial x}-\left(1+\frac{\nu^{2}}{x^{2}}\right)\right] \tilde{\mathrm{g}}_{\nu}\left(x \mid x^{\prime} ; E\right)} \\
& =-\frac{4}{x l \sin \alpha} \delta\left(x-x^{\prime}\right)
\end{aligned}
$$

where we introduced the new variable $x=k \rho$, with $k^{2}=\frac{4 E}{l \sin ^{2} \alpha}$ and $\nu^{2}=\frac{m^{2}}{\sin ^{2} \alpha}$. The solutions of the homogeneous equation are the modified Bessel functions $\mathrm{I}_{|\nu|}(x)$ and $\mathrm{K}_{|\nu|}(x)$. Then, the regularity of $\tilde{\mathrm{g}}_{\nu}\left(x \mid x^{\prime} ; E\right)$ at $x=0, x \rightarrow \infty$, the continuity at $x=x^{\prime}$ and the jump in the first derivative impose the solution

$$
\tilde{\mathrm{g}}_{\nu}\left(\rho \mid \rho^{\prime} ; E\right)=\frac{4}{l \sin \alpha} \mathrm{I}_{|\nu|}\left(k \rho_{<}\right) \mathrm{K}_{|\nu|}\left(k \rho_{>}\right) .
$$

Here $\rho_{<}$and $\rho_{>}$denote the smaller and the larger, respectively, between the variables $\rho$ and $\rho^{\prime}$; we have also used the fact that the Wronskian $\mathrm{W}$ of the modified Bessel functions is $\mathrm{W}\left[\mathrm{I}_{\nu}, \mathrm{K}_{\nu}\right](x)=-\frac{1}{x}$. Inserting the solution found back in Eq. (3.17) and performing the inverse Laplace transform using the formula [14, §5.16.56]

$$
\begin{gathered}
\mathcal{L}^{-1}\left\{\mathrm{~K}_{\nu}[(\sqrt{a}+\sqrt{b}) \sqrt{E}] \mathrm{I}_{\nu}[(\sqrt{a}-\sqrt{b}) \sqrt{E}]\right\}= \\
\frac{1}{2 L} e^{-\frac{a+b}{2 L}} \mathrm{I}_{\nu}\left(\frac{a-b}{2 L}\right) \quad(\Re a, \Re b>0),
\end{gathered}
$$

we arrive at the exact spectral expansion of $\mathrm{G}\left(\mathbf{R} \mid \mathbf{R}^{\prime} ; L\right)$ for a polymer on a cone: 


$$
\begin{aligned}
\mathrm{G}\left(\mathbf{R} \mid \mathbf{R}^{\prime} ; L\right) & =\frac{1}{\pi L l \sin \alpha} \sum_{m=-\infty}^{+\infty} \mathrm{I}_{\left|\frac{m}{\sin \alpha}\right|}\left(2 \rho \rho^{\prime} /\left(L l \sin ^{2} \alpha\right)\right) \\
& \times \exp \left[-\frac{\rho^{2}+\rho^{\prime 2}}{L l \sin ^{2} \alpha}\right] \exp \left[i m\left(\phi-\phi^{\prime}\right)\right], \quad(3.21)
\end{aligned}
$$

which is properly normalized to one $\left(\int d \mathbf{R} \mathrm{G}\left(\mathbf{R} \mid \mathbf{R}^{\prime} ; L\right)=\right.$ $1)$. For $\alpha=\frac{\pi}{2}$ we recover the expression in polar coordinates of the propagator of a Gaussian polymer chain in the plane [7,8].

As the total partition sum gives the area of the cone, we calculate the end-to-end distance by fixing $\mathbf{R}^{\prime}$ and integrating only over $\mathbf{R}$. Using cylindrical coordinates, with $\left(\mathbf{R}-\mathbf{R}^{\prime}\right)^{2}=\left(\rho^{2}+\rho^{\prime 2}\right) / \sin ^{2} \alpha+2 \rho \rho^{\prime}\left[1-\cos \left(\phi-\phi^{\prime}\right)\right]$ and applying various formulae [12 involving definite integrals of Bessel functions, we obtain

$$
\begin{aligned}
& \left\langle\left(\mathbf{R}-\mathbf{R}^{\prime}\right)^{2}\right\rangle=L l\left\{1+2 t^{2} \csc ^{2} \alpha\right. \\
& -\sqrt{\pi} \csc \alpha\left(1-\sin ^{2} \alpha\right){ }_{1} \mathrm{~F}_{1}\left(-1 / 2,1 ;-t^{2} \csc ^{2} \alpha\right) \\
& -2 \frac{\Gamma(3 / 2+1 / 2 \csc \alpha)}{\Gamma(1+\csc \alpha)}(\sin \alpha)^{(1-\csc \alpha)} t^{(1+\csc \alpha)} \\
& \left.\times{ }_{1} \mathrm{~F}_{1}\left[(\csc \alpha-1) / 2,1+\csc \alpha ;-t^{2} \csc ^{2} \alpha\right]\right\},
\end{aligned}
$$

where $t=\rho^{\prime} /(L l)^{1 / 2}$ and ${ }_{1} \mathrm{~F}_{1}$ is the confluent hypergeometric function.

Rather surprisingly, the mean-square distance depends on the position of the chain through the parameter $t$. To interpret this finding, we calculate the limiting values of $\left\langle\left(\mathbf{R}-\mathbf{R}^{\prime}\right)^{2}\right\rangle$ for $t \rightarrow \infty$ (polymer far on the conical surface or $\alpha \rightarrow 0$ ), $t \rightarrow 0$ (polymer with one end attached to the cone vertex) and $\alpha=\frac{\pi}{2}$ (polymer on a $2-D$ plane surface). One finds that

$$
\left\langle\left(\mathbf{R}-\mathbf{R}^{\prime}\right)^{2}\right\rangle=L l \text { for }\left\{t=0 ; t \rightarrow \infty ; \alpha=\frac{\pi}{2}\right\},
$$

which is just the Gaussian result in all cases. Physically, when the polymer chain is either fixed at the origin $(t=0)$ or far away from it $(t \rightarrow \infty)$, there are no other preferred points on the cone and $\left\langle\left(\mathbf{R}-\mathbf{R}^{\prime}\right)^{2}\right\rangle$ has the planar value (we should also remark that a cone is geometrically flat). In the crossover region, the singular character of the cone vertex becomes dominant and induces the contraction of the chain (possibly by winding about the origin). This behavior is illustrated in Fig. 2 and Fig. 3, where the mean-square distance $\left\langle\left(\mathbf{R}-\mathbf{R}^{\prime}\right)^{2}\right\rangle$ normalized at $L l$ is plotted as a function of the $t$ parameter and the angle $\alpha$.

\section{The Torus in $\mathbb{R}^{3}$}

Consider a torus embedded in $\mathbb{R}^{3}$, with the circular cross-section of radius $a$. The axial circle containing the centers of the circular sections has the radius $b>a$ (see
Fig. 11). The torus is centered about the $O z$ axis and symmetric with respect to the $x O y$ plane. We introduce the toroidal coordinates $\{\eta, \theta, \phi\},[15, \S 2.13]$ where $\phi$ is the usual azimuthal angle -in cylindrical coordinatesabout $O z$ :

$$
\begin{aligned}
& x=\frac{c \sinh \eta \cos \phi}{\cosh \eta-\cos \theta} ; \quad y=\frac{c \sinh \eta \sin \phi}{\cosh \eta-\cos \theta} ; \\
& z=\frac{c \sin \theta}{\cosh \eta-\cos \theta},
\end{aligned}
$$

where $0 \leq \eta<\infty, 0 \leq \theta<2 \pi$ and $0 \leq \phi<2 \pi$. The surface of the torus corresponds to a fixed value $\eta_{0}$ of the coordinate $\eta$. Then we have the following geometrical relations:

$$
b=c \operatorname{coth} \eta_{0} ; a=\frac{c}{\sinh \eta_{0}} ; c^{2}=b^{2}-a^{2} .
$$

The induced metric tensor is $g_{\theta \theta}=\frac{c^{2}}{\left(\cosh \eta_{0}-\cos \theta\right)^{2}}$; $g_{\phi \phi}=\frac{c^{2} \sinh ^{2} \eta_{0}}{\left(\cosh \eta_{0}-\cos \theta\right)^{2}} ; \quad g_{\theta \phi}=g_{\phi \theta}=0 ; \sqrt{g}=$ $\frac{c^{2} \sinh \eta_{0}}{\left(\cosh \eta_{0}-\cos \theta\right)^{2}}$. We recall that the length interval on the surface is $d s^{2}=g_{\theta \theta} d \theta^{2}+g_{\phi \phi} d \phi^{2}$ and the volume element is $d^{2} \mathbf{r}=\sqrt{g} d \theta d \phi$.

The calculation of the propagator of a Gaussian chain on a torus in $\mathbb{R}^{3}$ (which is equivalent to solving the problem of a free quantum particle moving on the same surface) cannot be done in closed form, as involves finding the eigenvalues of a Hill-type equation. Still, using the path-integral formalism and making certain "sloppy" approximations, we were able to derive an approximate form of $\mathrm{G}\left(\mathbf{R} \mid \mathbf{R}^{\prime} ; L\right)$ that behaves physically correct when computing moments of the distribution of $\mathbf{R}-\mathbf{R}^{\prime}$, which are the experimentally relevant quantities we are interested in. This approach will be justified a posteriori by showing that one recovers the usual flat surface case and the expected asymptotic regimes obtained for spheres and cylinders.

We start be recalling the path-representation (2.1) written in a compact form as

$$
\mathrm{G}\left(\mathbf{R} \mid \mathbf{R}^{\prime} ; L\right)=\int_{\mathbf{r}_{0}=\mathbf{R}^{\prime}}^{\mathbf{r}_{N}=\mathbf{R}} \mathcal{D}[\mathbf{r}] \exp \left(-\sum_{j=1}^{N} \mathrm{~S}_{j}\right)
$$

where the integration measure is given explicitly by

$$
\mathcal{D}[\mathbf{r}]=\lim _{\substack{N \rightarrow \infty \\ \delta s_{j} \rightarrow 0 \\ \sum_{j} \delta s_{j}=L}} \prod_{j=1}^{N} \frac{1}{\pi l \delta s_{j}} \prod_{j=1}^{N-1} d^{2} \mathbf{r}_{j}
$$

and $\mathrm{S}_{j}$ is the short-length polymer action

$$
\mathrm{S}_{j}=\frac{\left(\mathbf{r}_{j}-\mathbf{r}_{j-1}\right)^{2}}{l \delta s_{j}} \text {. }
$$


In the previous expressions, $\delta s_{j}=s_{j}-s_{j-1}$ (with $\left.j=\overline{1, N}, s_{0}=0, s_{N}=L\right)$ and $\mathbf{r}_{j}$ is the position vector about the origin of the $j$-th element of the discretized chain.

In order to evaluate the path-integral, due to the mentioned equivalence between the statistical mechanics of polymers and quantum mechanics, we will apply the method used to calculate the propagator of a free particle moving on a circle and in the presence of a ring-shaped defect [6, 8, 16].

We start with the action element $\mathrm{S}_{j}$. Using the length interval on the torus, we write $\mathrm{S}_{j}$ in a symmetrized form

$$
\begin{aligned}
\mathrm{S}_{j} & =\frac{c^{2}}{l} \frac{1}{\delta s_{j}\left(\cosh \eta_{0}-\cos \theta_{j}\right)\left(\cosh \eta_{0}-\cos \theta_{j-1}\right)} \\
& \times\left[\left(\Delta \theta_{j}\right)^{2}+\sinh ^{2} \eta_{0}\left(\Delta \phi_{j}\right)^{2}\right]
\end{aligned}
$$

where $\left\{\theta_{j}=\theta\left(s_{j}\right), \phi_{j}=\phi\left(s_{j}\right)\right\}$ are the toroidal coordinates of the element located at $s_{j}$ along the chain, $\Delta \theta_{j}=\theta_{j}-\theta_{j-1}$ and $\Delta \phi_{j}=\phi_{j}-\phi_{j-1}$.

Next we apply a local rescaling [0],17], which reads

$$
\sigma_{j}=\delta s_{j}\left(\cosh \eta_{0}-\cos \theta_{j}\right)\left(\cosh \eta_{0}-\cos \theta_{j-1}\right)
$$

and which satisfies the global scaling relation:

$$
\sigma=\sum_{j=1}^{N} \sigma_{j}=L\left(\cosh \eta_{0}-\cos \theta\right)\left(\cosh \eta_{0}-\cos \theta^{\prime}\right)
$$

This constraint is nontrivial, as it must be compatible with the local scaling (3.30). That it gives the correct answer when applied in the path integral and when one uses an anisometric discretization (unequal length intervals $\delta s_{j}$ ), was discussed by Inomata 17. Basically, it amounts to the prescription [18] of ignoring terms of $O\left(\delta s_{j}^{1+\epsilon}\right)$ in the path-integral, if $\epsilon>0$. Then, the discretized functional measure becomes:

$$
\begin{aligned}
\mathcal{D}[\mathbf{r}] & =\left(\cosh \eta_{0}-\cos \theta\right)\left(\cosh \eta_{0}-\cos \theta^{\prime}\right) \\
& \times \prod_{j=1}^{N} \frac{1}{\sigma_{j}} \prod_{j=1}^{N-1} d \theta_{j} d \phi_{j} c^{2} \sinh \eta_{0} .
\end{aligned}
$$

We apply now the length rescaling in the action $\mathrm{S}_{j}$ given by (3.29) directly, by dropping boldly any terms coming from a rigorous expansion of $\Delta \theta_{j}$ and $\Delta \phi_{j}$ about the new length variable $\sigma_{j}$. As mentioned, this will be justified by the final results. Combining then with the integration measure, we get the approximate discretized propagator $\mathrm{G}_{N}$

$$
\begin{array}{r}
\mathrm{G}_{N}\left(\mathbf{R} \mid \mathbf{R}^{\prime} ; \sigma\right) \approx \frac{1}{c^{2}}\left(\cosh \eta_{0}-\cos \theta\right)\left(\cosh \eta_{0}-\cos \theta^{\prime}\right) \\
\times \prod_{j=1}^{N} \frac{c^{2}}{\pi l \sigma_{j}} \int_{0}^{2 \pi} \prod_{j=1}^{N-1} d \theta_{j} \exp \left[-\frac{c^{2}}{l} \sum_{j=1}^{N} \frac{\left(\Delta \theta_{j}\right)^{2}}{\sigma_{j}}\right]
\end{array}
$$

$$
\begin{aligned}
& \times \int_{0}^{2 \pi} \prod_{j=1}^{N-1} d \phi_{j} \sinh \eta_{0} \\
& \times \exp \left[-\frac{c^{2} \sinh ^{2} \eta_{0}}{l} \sum_{j=1}^{N} \frac{\left(\Delta \phi_{j}\right)^{2}}{\sigma_{j}}\right]
\end{aligned}
$$

Observing that the integrals over the angular coordinates are similar to the path integral for a free particle moving on a circle (e.g. see Ref. [ [6,7]]), we have the formula

$$
\begin{array}{r}
\prod_{j=1}^{N}\left(\frac{\alpha}{\pi \sigma_{j}}\right)^{1 / 2} \int_{0}^{2 \pi} \prod_{j=1}^{N-1} d \psi_{j} e^{-\alpha \sum_{j=1}^{N} \frac{\left(\Delta \psi_{j}\right)^{2}}{\sigma_{j}}} \\
=\sqrt{\frac{\alpha}{\pi \sigma}} \sum_{n=-\infty}^{\infty} e^{-\frac{\alpha}{\sigma}\left(\psi-\psi^{\prime}+2 \pi n\right)^{2}} .
\end{array}
$$

Here $\alpha$ is an arbitrary positive constant and $\psi_{j}=\psi\left(\sigma_{j}\right)$ is the discretized angular variable on the circle, with $\psi^{\prime}$ and $\psi$ denoting the initial and the final positions. Equivalently, recognizing that the angular path integral contains trajectories that wind a different number of times around the origin, one can use the covering space mapping [6, 7].

Returning to Eq. 3.33) of the approximate discretized propagator $\mathrm{G}_{N}$, integrating over the angles using formula (3.34), and replacing $\sigma$ with its global value (3.31), we finally obtain - up to a normalization factor - the approximate distribution function of the end-to-end distance for a polymer chain on a curved torus in $\mathbb{R}^{3}$, expressed as a winding number expansion:

$$
\begin{aligned}
\mathrm{G}(\mathbf{R} & \left.\mid \mathbf{R}^{\prime} ; L\right) \approx \frac{1}{\pi L l} \sum_{n=-\infty}^{\infty} \sum_{m=-\infty}^{\infty} \exp \left\{-\frac{c^{2}}{L l}\right. \\
& \times \frac{1}{\left(\cosh \eta_{0}-\cos \theta\right)\left(\cosh \eta_{0}-\cos \theta^{\prime}\right)} \\
& \left.\times\left[\left(\theta-\theta^{\prime}+2 \pi n\right)^{2}+\left(\sinh ^{2} \eta_{0}\right)\left(\phi-\phi^{\prime}+2 \pi m\right)^{2}\right]\right\}
\end{aligned}
$$

Another compact and illuminating form is obtained by employing the transformations (3.12) and (3.13), which yield the angular momentum expansion

$$
\begin{aligned}
\mathrm{G}(\mathbf{R} & \left.\mid \mathbf{R}^{\prime} ; L\right) \approx \frac{1}{4 \pi^{2}} \frac{\left(\cosh \eta_{0}-\cos \theta\right)\left(\cosh \eta_{0}-\cos \theta^{\prime}\right)}{c^{2} \sinh \eta_{0}} \\
& \times \Theta_{3}\left[\left(\theta-\theta^{\prime}\right) / 2, i \sigma\left(\theta, \theta^{\prime}\right) l /\left(4 \pi c^{2}\right)\right] \\
& \times \Theta_{3}\left[\left(\phi-\phi^{\prime}\right) / 2, i \sigma\left(\theta, \theta^{\prime}\right) l /\left(4 \pi c^{2} \sinh ^{2} \eta_{0}\right)\right]
\end{aligned}
$$

where we recall that $\sigma\left(\theta, \theta^{\prime}\right)=L\left(\cosh \eta_{0}-\cos \theta\right)\left(\cosh \eta_{0}\right.$ $\left.-\cos \theta^{\prime}\right), c^{2}=b^{2}-a^{2}$ and $\cosh \eta_{0}=\frac{b}{a}$. $\Theta_{3}$ is the theta function defined as 12

$$
\Theta_{3}(z, \tau)=\sum_{n=-\infty}^{\infty} e^{i \pi \tau n^{2}} e^{2 i z n} \quad(\Im \tau>0)
$$


First, we stress that this is not the exact Green function of the heat equation on the curved torus. Still, as necessary, the expression is symmetric in $\mathbf{R}, \mathbf{R}^{\prime}$, translationally invariant with respect to the azimuthal angle $\phi$, and in the limit $\eta_{0} \rightarrow \infty \equiv a \rightarrow 0$ reduces properly to the probability distribution of the end-to-end vector for a polymer on a circle (see the angular part in Eq. (3.14)). Also, it obeys the initial condition

$$
\mathrm{G}\left(\mathbf{R} \mid \mathbf{R}^{\prime} ; 0\right)=\delta^{(2)}\left(\mathbf{R}-\mathbf{R}^{\prime}\right)
$$

and its trace has, at least numerically, the correct limit [19] when $\frac{L l}{a^{2}} \rightarrow 0$ :

$$
\begin{aligned}
\mathrm{Z}(x \rightarrow 0, t) & =\iint d^{2} \mathbf{R} \mathrm{G}(\mathbf{R}, L \mid \mathbf{R}, 0) \\
& \simeq 4 \pi \frac{t}{x}+\frac{\pi^{3}}{60} x \frac{t\left(t-\sqrt{t^{2}-1}\right)}{\sqrt{t^{2}-1}}+o\left(x^{2}\right)
\end{aligned}
$$

where we introduced the dimensionless variables:

$$
t=\cosh \eta_{0}=\frac{b}{a} ; \quad x=\frac{L l}{a^{2}}
$$

A closed formula for $\left\langle\left(\mathbf{R}-\mathbf{R}^{\prime}\right)^{2}\right\rangle$ cannot be readily obtained and a numerical calculation is required. To begin with, we write the distance (in $\mathbb{R}^{3}$ ) between the ends of the polymer chain:

$$
\begin{aligned}
\left(\mathbf{R}-\mathbf{R}^{\prime}\right)^{2} & =\frac{2 c^{2}}{\left(\cosh \eta_{0}-\cos \theta\right)\left(\cosh \eta_{0}-\cos \theta^{\prime}\right)}\left[\cosh ^{2} \eta_{0}\right. \\
& \left.-\sinh ^{2} \eta_{0} \cos \left(\phi-\phi^{\prime}\right)-\cos \left(\theta-\theta^{\prime}\right)\right] .
\end{aligned}
$$

Using the Green function (3.36) in the formula for the mean-square end-to-end distance (2.9) with the proper volume element on the surface of the torus, evaluating the integrals over the azimuthal angles $\phi$ and $\phi^{\prime}$ by applying the definition (3.37) of the theta function and the formula:

$$
\iint_{0}^{2 \pi} d \phi d \phi^{\prime} \cos \left(\phi-\phi^{\prime}\right) e^{i m\left(\phi-\phi^{\prime}\right)}=2 \pi^{2}\left(\delta_{-1 m}+\delta_{+1 m}\right)
$$

and expressing all quantities in terms of the dimensionless variables from Eq. (3.40), we eventually obtain the mean-square end-to-end distance as

$$
\begin{aligned}
& \frac{\left\langle\left(\mathbf{R}-\mathbf{R}^{\prime}\right)^{2}\right\rangle}{a^{2}}=\frac{2\left(t^{2}-1\right)}{N(x, t)} \iint_{0}^{2 \pi} d \theta d \theta^{\prime} \\
& \times \frac{t^{2}+\left(1-t^{2}\right) e^{-\frac{x(t-\cos \theta)\left(t-\cos \theta^{\prime}\right)}{4\left(t^{2}-1\right)^{2}}}-\cos \left(\theta-\theta^{\prime}\right)}{(t-\cos \theta)^{2}\left(t-\cos \theta^{\prime}\right)^{2}} \\
& \times \Theta_{3}\left[\frac{\theta-\theta^{\prime}}{2}, \frac{i x(t-\cos \theta)\left(t-\cos \theta^{\prime}\right)}{4 \pi\left(t^{2}-1\right)}\right],
\end{aligned}
$$

where $N(x, t)$ is given by

$$
\begin{aligned}
N(x, t)= & \iint_{0}^{2 \pi} d \theta d \theta^{\prime} \frac{1}{(t-\cos \theta)\left(t-\cos \theta^{\prime}\right)} \\
& \times \Theta_{3}\left[\frac{\theta-\theta^{\prime}}{2}, \frac{i x(t-\cos \theta)\left(t-\cos \theta^{\prime}\right)}{4 \pi\left(t^{2}-1\right)}\right] .
\end{aligned}
$$

The partition function $Z(x, t)$ of the polymer on the torus is:

$$
Z(x, t)=a^{2}\left(t^{2}-1\right)^{\frac{3}{2}} N(x, t)
$$

This reduces properly to the area $4 \pi^{2} a b$ of the torus when $x \gg 1, t \gg 1(\Theta(z, \tau) \simeq 1)$ :

$$
Z(x, t) \simeq 4 \pi^{2} a b \sqrt{1-\frac{1}{t^{2}}} \simeq 4 \pi^{2} a b
$$

Physically, for different values of $x$ and $t$, three regimes are expected:

$$
\begin{aligned}
& \text { a) } L l \ll a^{2}<b^{2} \Longleftrightarrow \frac{x}{t^{2}}<x \ll 1, \\
& \text { b) } a^{2} \ll L l \ll b^{2} \Longleftrightarrow \frac{x}{t^{2}} \ll 1 \ll x, \\
& \text { c) } a^{2}<b^{2} \ll L l \Longleftrightarrow 1 \ll \frac{x}{t^{2}}<x,
\end{aligned}
$$

where in the first case we should recover the solution for a polymer in the plane, in the second we must obtain the result for a polymer on a cylinder and in the third, the mean square end-to-end distance should reach a constant value (corresponding to a large winding number about both the $O z$ axis and the axial circle of radius $b$ ).

These regimes are manifest in Fig. A, where the results for the torus are plotted with thin lines, for $t=$ $\{2 ; 4 ; 6 ; 8 ; 10\}$.

Initially (the first regime in Eq. (3.47)), the polymer is too small to explore the geometry of the surface, and we recover (numerically) the case of a polymer in a plane. This is also the behavior found for the other surfaces.

As the length of the chain increases, it starts winding along the circle of radius $a$ and then along the axial circle, about the $O z$ axis. When $t$ increases, the behavior is similar - for a certain range of $x$ values - to that of a chain on a cylinder: the mean-square end-to-end distance is linear in $\mathrm{Ll} / 2$. We can check analytically that the approximate propagator from Eq. (3.36) produces the correct behavior. Because $a^{2} \ll L l \ll b^{2}$, the term $n=0$ dominates in the theta function in Eq. (3.43) $\left(\Theta_{3}(z, \tau) \approx 1\right)$ and the exponential coefficient of $1-t^{2}$ can be expanded, so one obtains explicitly

$$
\left\langle\left(\mathbf{R}-\mathbf{R}^{\prime}\right)^{2}\right\rangle \simeq 2 a^{2}+\frac{L l}{2} \quad\left(a^{2} \ll L l \ll b^{2}\right)
$$


which is just the limit for the polymer on the cylinder of radius $a$ from Eq. (3.15).

Eventually, at any given $t$ and large enough $x$, $\left\langle\left(\mathbf{R}-\mathbf{R}^{\prime}\right)^{2}\right\rangle$ departs from the behavior of a polymer on a cylinder and approaches a constant value, which is a function only of the geometrical parameters $a$ and $b$. This limiting value can be explicitly calculated. Now we have $a^{2}<b^{2} \ll L l$, thus once again $\Theta_{3}(z, \tau) \approx 1$ but the exponential coefficient of $1-t^{2}$ in the formula for distance (3.43) is zero, which gives the limit:

$$
\left\langle\left(\mathbf{R}-\mathbf{R}^{\prime}\right)^{2}\right\rangle \approx 2\left(a^{2}+b^{2}\right) \quad\left(a^{2}<b^{2} \ll L l\right) .
$$

If either $a$ or $b$ radii becomes 0 , one recovers the asymptotic limits of the end-to-end distance for a polymer on a circle or on a sphere, respectively, as found in Eq. (3.9).

Although the asymptotic behavior is correctly recovered, we expect our results in the crossover region of intermediate values of $t$ and $x$ to be only approximate.

\section{CONCLUSIONS}

Due to its relevance to studies of the behavior of polymers confined at interfaces, we have considered here the problem of a linear, Gaussian polymer chain embedded in the following surfaces: the $\mathrm{S}^{D-1}$ sphere in $D$ dimensions, the cylinder, the cone and the curved torus in $\mathbb{R}^{3}$.

We obtained closed formulas for the probability distribution function $\mathbf{G}\left(\mathbf{R} \mid \mathbf{R}^{\prime} ; L\right)$ of the end-to-end vector of the chain for the sphere, the cylinder and the cone and an approximate propagator for the torus. We calculated, analytically in the case of the sphere, the cylinder and the cone, and numerically for the torus, the meansquare end-to-end distance (in the embedding space) of the chain. As such, at least in the limiting regimes previously described, the results are also valid for a free quantum particle on a torus if one uses the mapping $\left\{L \rightarrow t-t^{\prime} ; \frac{2}{l} \rightarrow \frac{m}{i \hbar}\right\}$ (where $t$ and $t^{\prime}$ represent the final and initial time coordinates of the particle).

Our calculations demonstrate the role played by the geometry (curvature and shape) of the interface in controlling the size of the chain.

As the size of the confining surface decreases, the polymer size is determined by the parameters of the confining surface instead of chain length. The crossover between the free Gaussian chain limit and the confined limit is controlled by a characteristic geometrical localization area $A$. $A$ is proportional to $a^{2}$ for the cases of spheres and cylinders, and there are two characteristic areas proportional to $a^{2}$ and $b^{2}$ for the case of curved torus. For the cone, the localization area is variable and is determined by the position of the singular point (the vertex) about the chain and by the angle $\alpha$. Explicit closed formulas are derived for the dependence of the mean-square end-to-end distance of a Gaussian chain on the geometrical parameters of the confining surface. The formulas for spheres and cylinders are simple (Eqs. (3.9,3.15)), describing the crossover between free polymer regime and confined polymer regime. For the case of a cone, the final expression is more complicated, but still exact. For a torus, there are three asymptotic regimes as outlined by Eq. (3.47), and the crossover behavior is obtained numerically from Eq. (3.43).

A number of open problems come into attention: the calculation of the mean-square geodesic end-to-end distance (as measured on the surface and not in the embedding space), the statistics of the winding numbers (mainly for the polymer on the torus case), the influence of the topological defects existing on the surface, the presence of excluded volume and other types of potential interactions. We hope to address some of these issues in the near future.

\section{ACKNOWLEDGMENTS}

Acknowledgment is made to the CUMIRP and Materials Research Science and Engineering Center at the University of Massachusetts, and the NSF Grant DMR 9625485. The authors are grateful to E. Cattani, J. Douglas, J. Machta and F. Pedit for useful discussions.

* Also at The National Institute of Materials Physics, Bucureşti, Romania.

[1] B. Alberts, D. Bray, J, Lewis, M. Raff, K. Roberts and J. D. Watson, Molecular Biology of the Cell (Garland Publishing, Inc. New York, 1994).

[2] L. S. Schulman, Techniques and Applications of Path Integration (John Wiley \& Sons, London, New York, 1981).

[3] G. Blatter, M. V. Feigel'man, V. B. Geshkenbein, A. I. Larkin and V. Vinokur, Rev. Mod. Phys. 66, 1125 (1994).

[4] M. Doi and S. F. Edwards, The Theory of Polymer Dynamics (Oxford Science Publications, New York, 1986).

[5] K. F. Freed, Adv. Chem. Phys. 22, 1 (1972).

[6] H. Kleinert, Path Integrals in Quantum Mechanics, Statistics and Polymer Physics, 2nd ed. (World Scientific, Singapore, 1995).

[7] D. C. Khandekar, S. V. Lawande and K. V. Bhagwat, Path-Integral Methods and their Applications (World Scientific, Singapore, 1993).

[8] F. Wiegel, Introduction to Path-Integral Methods in Physics and Polymer Science (World Scientific, 1986).

[9] C. Grosche and F. Steiner, Path Integrals on Curved Manifolds, Z. Phys. C-Particles and Fields 36, 699 (1987). 
[10] A. Erdelyi, W. Magnus, F. Oberhettinger, F. G. Tricomi, Higher Transcendental Functions, Vol. II (McGraw Hill, New York, 1985).

[11] C. Itzykson, Commun. Math. Phys. 36, 19 (1974).

[12] I. S. Gradshteyn and I. M. Ryzhik, Tables of Integrals, Series and Products, 5th ed., (Academic Press, London, New York, 1994).

[13] R. Courant and D. Hilbert, Methods of Mathematical Physics, Vol. I (John Wiley \& Sons, London, New York, 1989).

[14] A. Erdelyi, W. Magnus, F. Oberhettinger, F. G. Tricomi, Tables of Integral Transforms, Vol. I (McGraw Hill, New
York, 1954).

[15] G. Arfken, Mathematical Methods for Physicists, 2nd ed., (Academic Press, London, New York, 1970).

[16] R. C. Ramos Jr., C. C. Bernido and M. V. CarpioBernido, J. Phys. A: Math. Gen. 27, 8251 (1994).

[17] A. Inomata in Path Integrals from meV to MeV, 433, M. C. Gutzwiller et al. (eds.) (World Scientific, Singapore, 1986).

[18] R. P. Feynman and A. R. Hibbs, Quantum Mechanics and Path Integrals (McGraw-Hill, new York, 1965).

[19] H. P. McKean and I. M. Singer, J. Diff. Geom. 1, 43 (1967).

FIG. 1. The geometrical data that characterize the sphere (A), the cylinder (B), the cone (C) and the torus (D): $a$ is the radius of the sphere, of the cylinder and of the circular cross-section of the torus; $b$ is the radius of the axial circle of the torus; $\alpha$ is the angle of the cone at the vertex.

FIG. 2. The mean-square end-to-end distance $\left\langle\left(\mathbf{R}-\mathbf{R}^{\prime}\right)^{2}\right\rangle$ normalized to $L l$ for a Gaussian polymer chain embedded on a cone, as a function of $t=\frac{\rho^{\prime}}{\sqrt{L l}}$ and $\alpha$.

FIG. 3. The mean-square end-to-end distance $\left\langle\left(\mathbf{R}-\mathbf{R}^{\prime}\right)^{2}\right\rangle$ normalized to $L l$ for a Gaussian polymer chain embedded on a cone, as a function of $t=\frac{\rho^{\prime}}{\sqrt{L l}}$ and $\alpha: 3-D$ representation.

FIG. 4. The mean-square end-to-end distance $\left\langle\left(\mathbf{R}-\mathbf{R}^{\prime}\right)^{2}\right\rangle$ normalized to $a^{2}$ for a Gaussian polymer chain embedded on different surfaces, as a function of $\frac{L l}{a^{2}}$ and $t=\frac{b}{a}$. The symbols joined by thin lines represent the data for the polymer on the surface of the curved torus in $\mathbb{R}^{3}$ : thick solid line, the cylinder in $\mathbb{R}^{3}$; ○—o torus, $t=10 ; \square-\square$ torus, $t=8$; $\diamond-\diamond$ torus, $t=6$; $\triangle \longrightarrow \triangle$ torus, $t=4 ; \nabla-\nabla$ torus, $t=2$; thick dotted line, the sphere $\mathrm{S}^{D-1}$ in $\mathbb{R}^{D}$. 


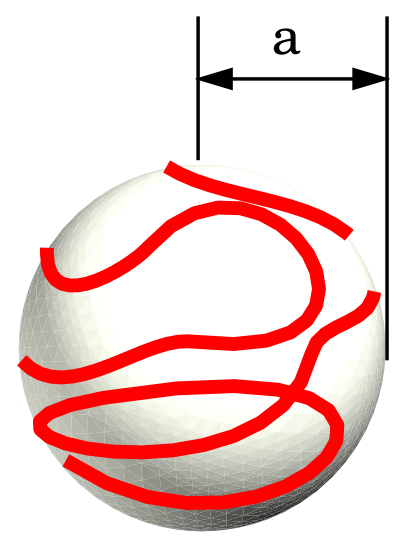

A.

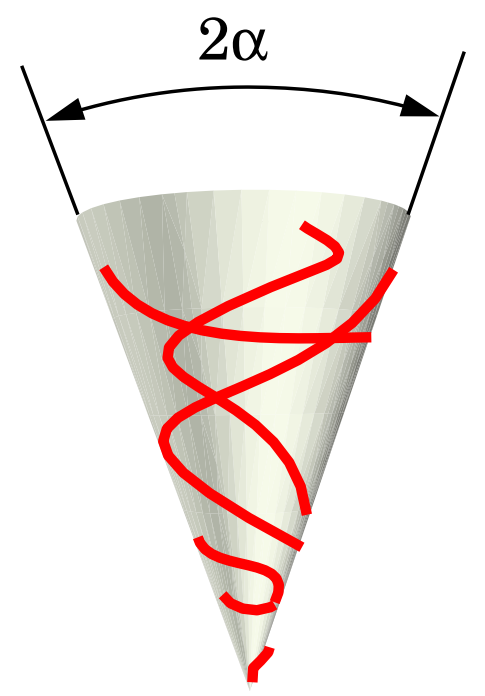

C.



B.



D.

Fig. 1 (Brownian Motion and Polymer Statistics on Certain Curved Manifolds Mondescu \& Muthukumar) 
Fig. 2 (Brownian Motion and Polymer Statistics on Certain Curved Manifolds - Mondescu/Muthukumar)

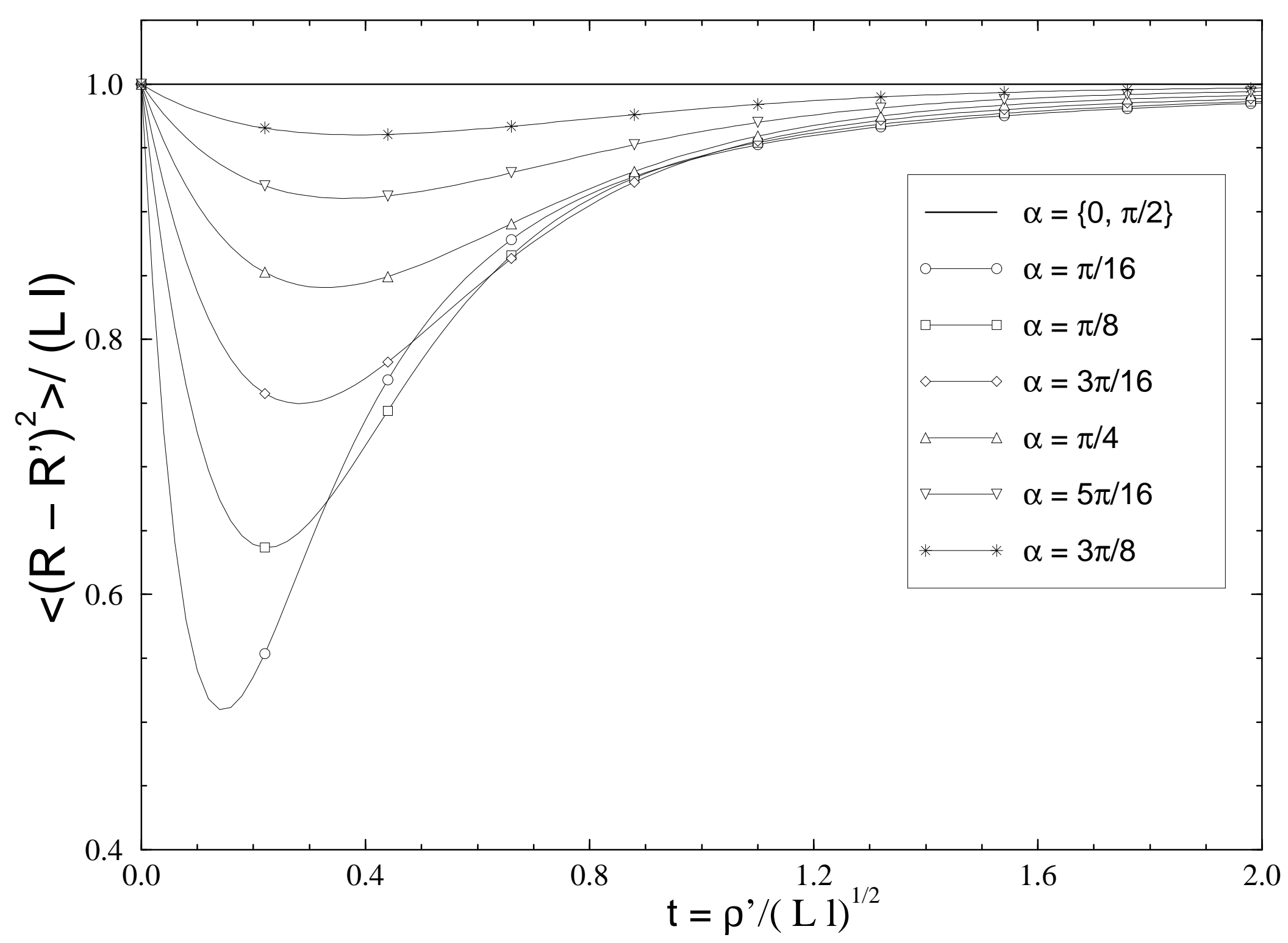




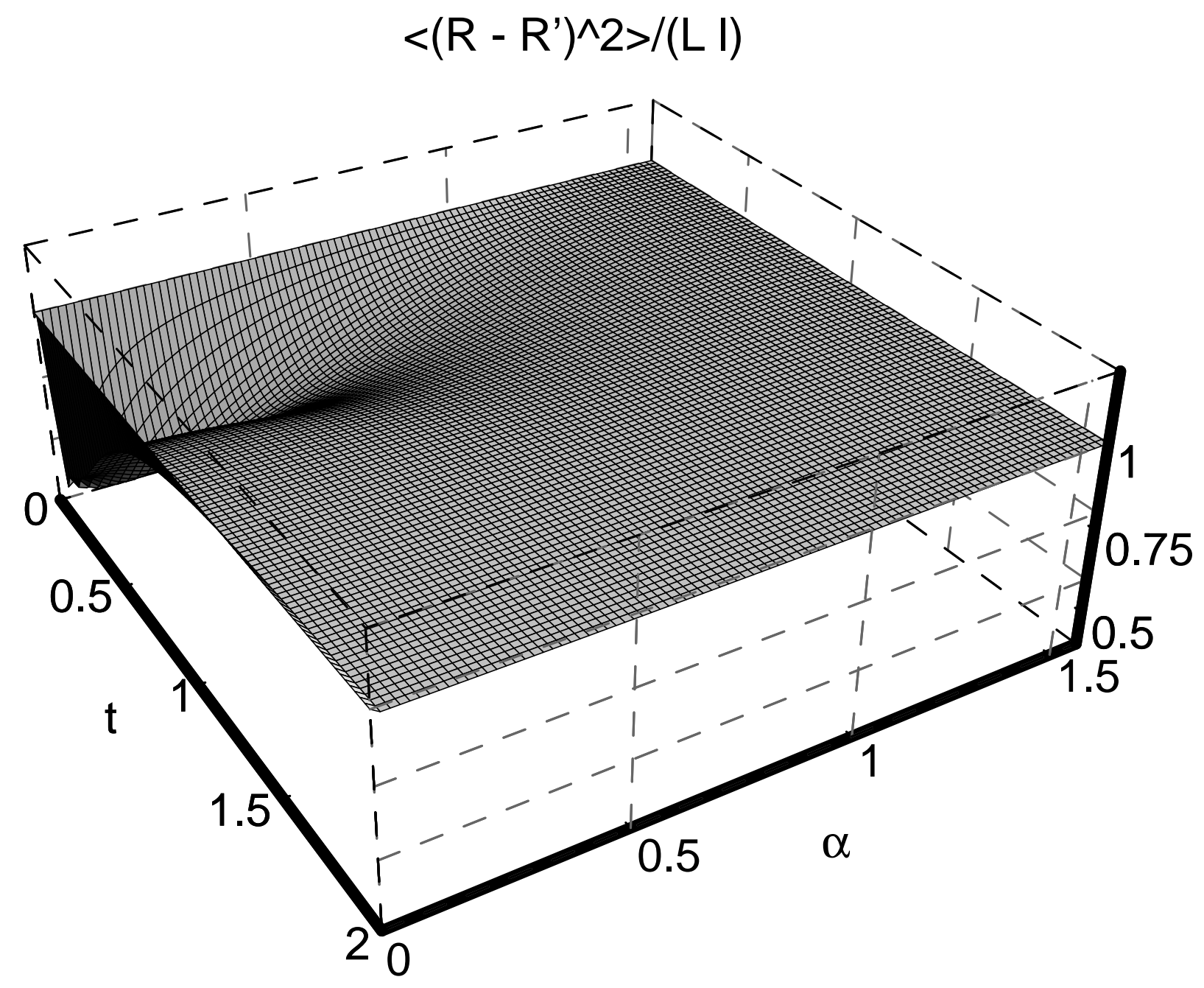


Fig.4 Brownian Motion and Polymer Statistics on Certain Curved Manifolds: Mondescu/Muthukumar

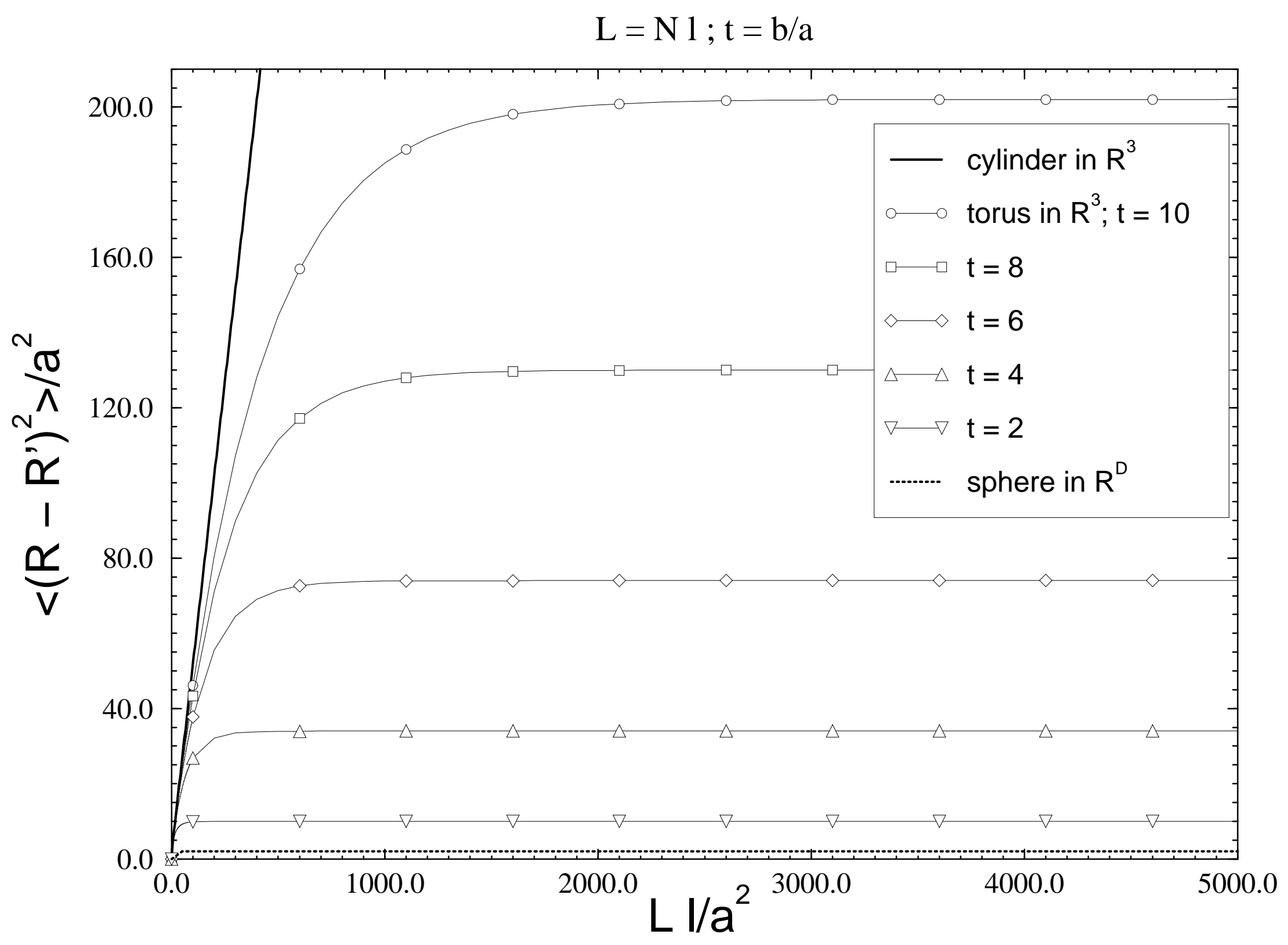

